\title{
Neural Correlates of Anesthesia in Newborn Mice and Humans
}

\author{
Mattia Chini ${ }^{1}$, Sabine Gretenkord ${ }^{1}$, Johanna K. Kostka ${ }^{1}$, Jastyn A. Pöpplau ${ }^{1}$, \\ Laura Cornelissen ${ }^{2,3}$, Charles B. Berde ${ }^{2,3}$, Ileana L. Hanganu-Opatz ${ }^{1 * t}$ \\ and Sebastian H. Bitzenhofer ${ }^{1 * \dagger \neq}$
}

\begin{abstract}
'Developmental Neurophysiology, Institute of Neuroanatomy, University Medical Center Hamburg-Eppendorf, Hamburg, Germany, ${ }^{2}$ Department of Anesthesiology, Critical Care and Pain Medicine, Boston Children's Hospital, Boston, MA, United States, ${ }^{3}$ Department of Anesthesia, Harvard Medical School, Boston, MA, United States
\end{abstract}

\section{OPEN ACCESS}

Edited by:

Tommaso Pizzorusso,

University of Florence, Italy

Reviewed by:

Paola Binda,

University of Pisa, Italy

Laura Baroncelli,

Italian National Research Council

(CNR), Italy

${ }^{*}$ Correspondence: Ileana L. Hanganu-Opatz hangop@zmnh.uni-hamburg.de Sebastian H. Bitzenhofer sbitzenhofer@ucsd.edu

${ }^{\dagger}$ These authors have contributed equally to this work

‡Present address: Sebastian H. Bitzenhofer, Center for Neural Circuits and

Behavior, Department of Neurosciences, University of California, San Diego, La Jolla, CA, United States

Received: 31 January 2019 Accepted: 03 May 2019

Published: 22 May 2019

Citation:

Chini M, Gretenkord S, Kostka JK, Pöpplau JA, Cornelissen L, Berde CB, Hanganu-Opatz IL and Bitzenhofer SH (2019) Neural Correlates of Anesthesia in Newborn Mice and Humans. Front. Neural Circuits 13:38. doi: 10.3389/fncir.2019.00038
Monitoring the hypnotic component of anesthesia during surgeries is critical to prevent intraoperative awareness and reduce adverse side effects. For this purpose, electroencephalographic (EEG) methods complementing measures of autonomic functions and behavioral responses are in use in clinical practice. However, in human neonates and infants existing methods may be unreliable and the correlation between brain activity and anesthetic depth is still poorly understood. Here, we characterized the effects of different anesthetics on brain activity in neonatal mice and developed machine learning approaches to identify electrophysiological features predicting inspired or end-tidal anesthetic concentration as a proxy for anesthetic depth. We show that similar features from EEG recordings can be applied to predict anesthetic concentration in neonatal mice and humans. These results might support a novel strategy to monitor anesthetic depth in human newborns.

Keywords: development, anesthesia, LFP, EEG, mouse, human, machine learning, network dynamics

\section{INTRODUCTION}

Reliable monitoring of anesthesia depth is critical during surgery. It allows for loss of consciousness, analgesia and immobility without incurring the risk of side effects and complications due to anesthetic misdosing. Typically used measures to monitor anesthesia depth are inspired and end-tidal anesthetic concentrations as well as physiologic parameters, including respiratory rate and depth (in the absence of neuromuscular blockade or controlled ventilation), heart rate, blood pressure, and responses to noxious stimuli (Punjasawadwong et al., 2014). These measures all respond to spinal and brainstem reflexes and are not specific for arousal or cortical responses to noxious events.

Anesthesia-induced changes in brain activity can be measured with electroencephalographic (EEG) recordings. Specific algorithms have been developed to predict anesthesia depth in adults (Glass et al., 1997; Prichep et al., 2004; Kreuzer, 2017). The most commonly used of such methods, the Bispectral Index, has been shown to significantly reduce intraoperative awareness, amount of anesthetic used, recovery time and post-anesthesia care unit stay in a recent Cochrane meta-analysis (Punjasawadwong et al., 2014), but see (Kalkman et al., 2011; Hajat et al., 2017). However, evidence of similar benefits in infants and younger children is sparse, as recently shown (Cornelissen et al., 2015, 2017, 2018a). EEG in anesthetized infants changes dramatically depending on postnatal age. Slow oscillations are present from birth on, whereas theta and alpha oscillations 
occur 3 months after birth, but lack the frontal dominance typically seen in adults (Davidson et al., 2005; Lo et al., 2009; Hayashi et al., 2012; Cornelissen et al., 2015; Koch et al., 2017).

EEG recordings mainly monitor neocortical activity. Converging evidence from animal and human studies has shown that most anesthetics slow EEG oscillations (Alkire et al., 2008; Chauvette et al., 2011; Purdon et al., 2015). While power at high frequency oscillations is reduced $(>40 \mathrm{~Hz})$, power at slower frequencies ( $<15 \mathrm{~Hz}$ ) is enhanced (Purdon et al., 2015). The computations underlying proprietary indexes such as the Bispectral index or Narcotrend are thought to take advantage of these phenomena (Kertai et al., 2012). However, in preterm and term neonates for the first weeks of life, EEG during sleep-wake cycles is weakly correlated with behavioral states and shows characteristic bursts or spontaneous activity transients (Milh et al., 2007; O'Toole et al., 2016). Anesthesia-induced theta and alpha oscillations have been reported to emerge around 3-4 months of age, albeit with less frontal predominance than in older children and adults (Cornelissen et al., 2015, 2018a). Moreover, high concentrations/doses of anesthetics have been reported to depress brain activity and enhance signal discontinuity in both human and rodent neonates (Chang et al., 2016; Cornelissen et al., 2017; Stolwijk et al., 2017). However, to our knowledge, a comprehensive algorithmic approach identifying EEG parameters that robustly correlate with anesthetic depth during early postnatal development is still lacking.

Here, we developed a novel strategy to model anesthesia depth by using common electrophysiological features that correlate with inhaled anesthetic concentrations during early development of mice and humans at similar stage of brain development. We performed intracranial electrophysiological recordings to study the temporal and dose-dependent dynamics of brain activity in neonatal mice [postnatal day (P) 8-10] during bolus urethane administration, and during dose-titrated isoflurane general anesthesia, respectively. Dominant local field potential (LFP) features of anesthetic state were identified and used to develop a machine-learning algorithm that distinguishes non-anesthetized from deeply anesthetized states and predicts anesthetic concentration as a proxy for anesthetic depth. Using a similar approach, we used multielectrode EEG recordings to study the dose-dependent dynamics of brain activity in a secondary analysis of a combined new and previously reported data set (Cornelissen et al., 2018a) of human infants 0-6 months of age during induction, maintenance and emergence from general anesthesia (sevoflurane, isoflurane, or desflurane) administered for routine surgical care. Dominant EEG features of anesthetic state were identified and used to develop a machinelearning algorithm to predict end-tidal volume anesthetic concentration (an indirect measure of anesthetic concentration in the brain, and anesthetic depth).

\section{MATERIALS AND METHODS}

\section{Animals}

All experiments were performed in compliance with the German laws and the guidelines of the European Community for the use of animals in research and were approved by the local ethical committee (G132/12, G17/015, N18/015). Experiments were carried out on $\mathrm{C} 57 \mathrm{Bl} / 6 \mathrm{~J}$ mice of both sexes. Timedpregnant mice from the animal facility of the University Medical Center Hamburg-Eppendorf were housed individually at a $12 \mathrm{~h}$ light/12 h dark cycle, with ad libitum access to water and food. Day of birth was considered P0.

\section{In vivo Electrophysiology in Neonatal Mice}

Multisite extracellular recordings were performed in the prefrontal cortex (PFC) and HP, or lateral entorhinal cortex (LEC) and olfactory bulb (OB) of P8-10 mice. Pups were on a heating blanket during the entire procedure. Under isoflurane anesthesia (induction: 5\%; maintenance: $2.5 \%$ ), craniotomies were performed above PFC $(0.5 \mathrm{~mm}$ anterior to Bregma, 0.1-0.5 $\mathrm{mm}$ right to Bregma) and $\mathrm{HP}$ (3.5 $\mathrm{mm}$ posterior to Bregma, $3.5 \mathrm{~mm}$ right to Bregma), or LEC (0 $\mathrm{mm}$ anterior to lambda, $6.5 \mathrm{~mm}$ right to lambda) and $\mathrm{OB}(0.5-0.8 \mathrm{~mm}$ anterior from the frontonasal suture, $0.5 \mathrm{~mm}$ right from internasal suture). Pups were head-fixed into a stereotaxic apparatus using two plastic bars mounted on the nasal and occipital bones with dental cement. Multisite electrodes (NeuroNexus, MI, USA) were inserted into PFC (four-shank, A4x4 recording sites, $100 \mu \mathrm{m}$ spacing, $2.0 \mathrm{~mm}$ deep) and HP (one-shank, A1x16 recording sites, $50 \mu \mathrm{m}$ spacing, $1.6 \mathrm{~mm}$ deep, $20^{\circ}$ angle from the vertical plane), or LEC (one-shank, A1x16 recording sites, $100 \mu \mathrm{m}$ spacing, $2 \mathrm{~mm}$ deep, $10^{\circ}$ angle from the vertical plane) and $\mathrm{OB}$ (one-shank, A1x16 recording sites, $50 \mu \mathrm{m}$ spacing, 1.4-1.8 mm deep). A silver wire was inserted into the cerebellum and served as ground and reference electrode. Pups were allowed to recover for $30 \mathrm{~min}$ prior to recordings. Extracellular signals were band-pass filtered $(0.1-9,000 \mathrm{~Hz})$ and digitized $(32 \mathrm{kHz})$ with a multichannel extracellular amplifier (Digital Lynx SX, Neuralynx, Bozeman, MO, USA).

\section{In vivo Electrophysiology in Juvenile Mice}

Multisite extracellular recordings were performed in the PFC of P24-39 mice. Under isoflurane anesthesia (induction: 5\%; maintenance: $2.5 \%$ ), a metal head-post (Luigs and Neumann) was attached to the skull with dental cement and 2-mm craniotomies were performed above PFC (0.5-2.0 mm anterior to Bregma, 0.1-0.5 mm right to Bregma) and protected by a customized synthetic window. A silver wire was implanted in the cerebellum as ground and reference electrode. Surgery was performed at least 5 days before recordings. After recovery mice were trained to run on a custom-made spinning-disc. For recordings, craniotomies were uncovered and multisite electrodes (NeuroNexus, MI, USA) were inserted into PFC (oneshank, A1x16 recording sites, $50 \mu \mathrm{m}$ spacing, $2.0 \mathrm{~mm}$ deep). Extracellular signals were band-pass filtered $(0.1-9,000 \mathrm{~Hz})$ and digitized $(32 \mathrm{kHz})$ with a multichannel extracellular amplifier (Digital Lynx SX, Neuralynx, Bozeman, MO, USA).

\section{Recordings Under Urethane}

Activity was recorded for $15 \mathrm{~min}$ without anesthesia before intraperitoneally injecting urethane $(1 \mathrm{mg} / \mathrm{g}$ body weight; SigmaAldrich, St. Louis, MO, USA). Activity was recorded for further $45 \mathrm{~min}$. Animals were transcardially perfused after recordings, 
brains were sectioned coronally, and wide field images were acquired to verify recording electrode positions.

\section{Recordings Under Isoflurane}

Mouth piece of an isoflurane evaporator (Harvard Apparatus, Holliston, MA, USA) was placed in front of the pups in the recording setup until animals accustomed to it. Activity was recorded for $15 \mathrm{~min} 0 \%$ isoflurane, but with the evaporator running $(1.4 \mathrm{l} / \mathrm{min})$. Afterward, isoflurane was added to the airflow and increased every $15 \mathrm{~min}(1 \%, 2 \%, 3 \%)$. Animals were transcardially perfused after recordings, brains were sectioned coronally, and wide field images were acquired to verify recording electrode positions.

\section{Electroencephalographic Recordings in Human Neonates and Young Infants}

Neonates and infants who were scheduled for an elective surgical procedure were recruited from the pre-operative clinic at Boston Children's Hospital from 12/2012 to 08/2018 (under Institutional Review Board P-3544, with written informed consent obtained from parents/legal guardians). Subjects required surgery below the neck, were clinically stable on the day of study and American Society of Anesthesiologists' physical status I or II. Exclusion criteria were born with congenital malformations or other genetic conditions thought to influence brain development, diagnosed with a neurological or cardiovascular disorder, or born at $<32$ weeks post-menstrual age. Datasets from previously published work ( $n=25$; Cornelissen et al., 2018a) and new subjects $(n=10)$ were included in the analysis. Data are presented from 35 subjects aged 0-6 months.

\section{Anesthetic Management}

Each patient received anesthesia induced with sevoflurane (32 subjects), isoflurane (two subjects) or desflurane (one subject) alone or a combination of one of the previous and nitrous oxide. Epochs used for analysis were comprised of sevoflurane, isoflurane or desflurane administration with air and oxygen, titrated to clinical signs; end-tidal anesthetic concentration was adjusted per the anesthetist's impression of clinical need, not a pre-set end-tidal anesthetic concentration.

\section{EEG Recording}

EEG data were acquired using an EEG cap (WaveGuard EEG cap, Advanced NeuroTechnology, Netherlands). Thirty-threeor 41-recording electrodes were positioned per the modified international 10/20 electrode placement system. Reference and ground electrodes were located at $\mathrm{Fz}$ and $\mathrm{AFz}$ respectively. EEG activity from 0.1 to $500 \mathrm{~Hz}$ was recorded with an Xltek EEG recording system (EMU40EX, Natus Medical Inc., Canada). Signals were digitized at a sampling rate of $1,024 \mathrm{~Hz}$ and a resolution of 16-bit. The EEG recording was started prior to anesthetic induction to capture the loss of consciousness and stopped once the participant reached the Post-Anesthesia Recovery to capture recovery of consciousness. For some infants, the EEG recording was started after anesthetic induction.

\section{Clinical Data Collection}

Demographics and clinical information were collected from the electronic medical records and from the in-house Anesthesia
Information Management System (AIMS; Supplementary Table S1). None of the pathologies causing the need of surgery presented a risk for brain maturation. End-tidal sevoflurane, oxygen, and nitrous oxide concentrations were downloaded from the anesthetic monitoring device (Dräger Apollo, Dräger Medical Inc., Telford, PA, USA) to a recording computer in real-time using ixTrend software (ixcellence, Germany). Signals were recorded at a $1 \mathrm{~Hz}$ sampling rate.

\section{Data Analysis}

In vivo data were analyzed with custom-written algorithms in the Matlab environment. Data were processed as following: band-pass filtered (500-5,000 Hz) to analyze multi-unit activity (MUA) and band-pass filtered $(2-100 \mathrm{~Hz})$ using a third-order Butterworth filter before downsampling to analyze LFP. Filtering procedures were performed in a phase preserving manner.

\section{Multi-Unit Activity}

MUA was detected as the peak of negative deflections exceeding five times the standard deviation of the filtered signal and having a prominence larger than half the peak itself. Firing rates were computed by dividing the total number of spikes by the duration of the analyzed time window.

\section{Detection of Oscillatory Activity}

Discontinuous active periods were detected with a modified version of a previously developed algorithm for unsupervised analysis of neonatal oscillations (Cichon et al., 2014). Briefly, deflections of the root mean square of band-pass filtered signals $(1-100 \mathrm{~Hz})$ exceeding a variance-depending threshold were considered as network oscillations. The threshold was determined by a Gaussian fit to the values ranging from 0 to the global maximum of the root-mean-square histogram. If two oscillations occurred within $200 \mathrm{~ms}$ of each other they were considered as one. Only oscillations lasting $>1 \mathrm{~s}$ were included, and their occurrence, duration and amplitude were computed.

\section{Power Spectral Density}

For power spectral density analysis, 1 s-long windows of full signal or network oscillations were concatenated, and the power was calculated using Welch's method with non-overlapping windows.

\section{Imaginary Coherence}

The imaginary part of coherence, which is insensitive to volumeconduction-based effects (Nolte et al., 2004), was calculated by taking the absolute value of the imaginary component of the normalized cross-spectrum.

\section{Pairwise Phase Consistency}

Pairwise phase consistency (PPC) was computed as previously described (Vinck et al., 2010). Briefly, the phase in the band of interest was extracted using Hilbert transform and the mean of the cosine of the absolute angular distance among all pairs of phases was calculated.

\section{1/f Slope}

1/f slope was computed as previously described (Gao et al., 2017). We used robust linear regression (MATLAB function robustfit) 
to find the best fit over $20-40 \mathrm{~Hz}$ frequency range of the power spectral density, in 1-min bins.

\section{Sample Entropy}

Sample Entropy was computed using the SampEn function (MATLAB File Exchange) in $1.5 \mathrm{~s}$ windows and in $2 \mathrm{~Hz}$ frequency bins. Tolerance was set to $0.2 *$ std (signal), and tau to 1.

\section{EEG Data Analysis}

EEG signal was visually inspected to detect and reject channels with low signal to noise ratio and re-referenced to a common average reference. The signal was automatically scored in $5 \mathrm{~s}$ epochs, and channels in which signal was significantly contaminated by artifacts (patient handling, surgical electrocautery etc.) were discarded. Epochs were rejected if signal was saturated due to electrocautery, signal exceeded $150 \mu \mathrm{V}$, or the median signal across all EEG channels exceeds $30 \mu \mathrm{V}$ (Supplementary Figure S5). Minutes containing more than $10 \mathrm{~s}$ of contaminated signal were removed from further analysis. On average $14 \pm 9 \%$ (median \pm median absolute deviation) of the signal was discarded. To compute EEG amplitude, we smoothed the absolute value of the signal, using a moving average filter with a window of 1,024 points ( $1 \mathrm{~s})$. If more than one volatile anesthetic was used, we retained only epochs in which the main anesthetic was used in isolation. Subjects with epidural anesthesia halfway through the surgery ( $n=2$ subjects), or with less than 20 min of artifact-free signal ( $n=5$ subjects) were excluded from further analysis.

\section{Feature Engineering}

Features to predict anesthetic concentration in neonatal mice were calculated in 1-min bins. LFP power in the $1-100 \mathrm{~Hz}$ range in $10 \mathrm{~Hz}$ bins, the percentage of active periods, median length and number of oscillations, median and maximum signal amplitude were computed. All features were computed for both PFC and HP and were normalized to their median value in the non-anesthetized $15 \mathrm{~min}$ of recordings. Features to predict anesthetic concentration in human infants were also calculated in 1-min bins. The median amplitude of the smoothed EEG signal and the percentage of the EEG envelope that fell into each amplitude quartile was computed. Amplitude quartiles were computed on the entire EEG trace, averaged over channels. All features were calculated for unfiltered signal, and in the $1-50 \mathrm{~Hz}$ range in $5 \mathrm{~Hz}$ bins, averaged over channels. Features were normalized to their median value in the non-anesthetized portion of the recording, or lowest anesthetic concentration, if no artifact-free minute was available.

\section{Regressors}

Machine-learning analyses were performed using Python (Python Software Foundation, NH, USA) in the Spyder (Pierre Raybaut, The Spyder Development Team) development environment. Model training and performance evaluation were carried out using the scikit-learn toolbox. The set was iteratively $(n=100)$ divided between a training $(2 / 3$ of the set) and a crossvalidation (1/3) set. Hyper-parameter of the model was tuned on the training set, which was further split using the standard three-fold cross-validation split implemented by the function "GridSearchCV," to which a "pipeline" object was passed. The "pipeline" object was built using the "Pipeline" function, and concatenating quantile transformation of the input features ("Quantile Transformer," tuning the number of quantiles), feature selection ("Select Percentile," using mutual information and tuning the percentage of features to select) and Radial Basis Function (RBF) kernel support-vector classification/regression (tuning the regularization parameters $\mathrm{C}$ and epsilon (regression only), and the kernel coefficient gamma). The classifier input was fed to the regressor as an additional feature. Performance assessment was then computed on the cross-validation set. Regressor decision space was reduced and plotted with $t$-sne. The decision space was approximated by imposing a Voronoi tessellation on the $2 \mathrm{~d}$ plot, using k-nearest regression on the t-sne coordinates (Migut et al., 2015).

\section{Statistics}

Statistical analyses were performed using R Statistical Software (Foundation for Statistical Computing, Austria). Data were tested for significant differences $\left({ }^{*} p<0.05,{ }^{* *} p<0.01\right.$ and $* * * p<0.001)$ using non-parametric one- and two-way repeatedmeasures ANOVA (ARTool $\mathrm{R}$ package) with Bonferroni corrected post hoc analysis (emmeans $\mathrm{R}$ package). Correlations were computed using Spearman's rank correlation coefficient (rho). No statistical measures were used to estimate the sample size since the effect size was unknown. For the main experimental results, more information about tests used, values and parameters are provided in the supplementary material (Supplementary Table S2).

\section{Data Availability}

Electrophysiological data for hippocampus and prefrontal cortex mouse recordings, under both urethane and isoflurane condition, is available at the following openaccess repository: https://web.gin.g-node.org/mchini/Neural_ correlates_of_anesthesia_in_newborn_mice_and_humans.

\section{RESULTS}

\section{Anesthesia Affects the Occurrence but Not the Spectral and Temporal Structure of Oscillatory Events in Neonatal Mice}

We monitored the impact of anesthesia on immature brain activity in several cortical areas [PFC, hippocampus (HP), and LEC] as well as in a sensory area OB. For this, multi-site extracellular recordings of LFP and MUA were performed from P8 to 10 mice before and for $45 \mathrm{~min}$ after induction of anesthesia by intraperitoneal urethane injection (Figure 1A), an anesthetic commonly used in rodents (Khazipov et al., 2004; Colonnese and Khazipov, 2010).

The recorded network activity had a highly fragmented structure (defined as discontinuous activity) in all investigated areas (PFC, HP, LEC and OB). The full signal (i.e., entire LFP trace) consisted of transient episodes of oscillatory discharges with mixed frequencies (from here referred to as "active periods"), alternating with periods of relative electrical silence 
A A Urethane ( $1 \mathrm{mg} / \mathrm{g}$ body weight)
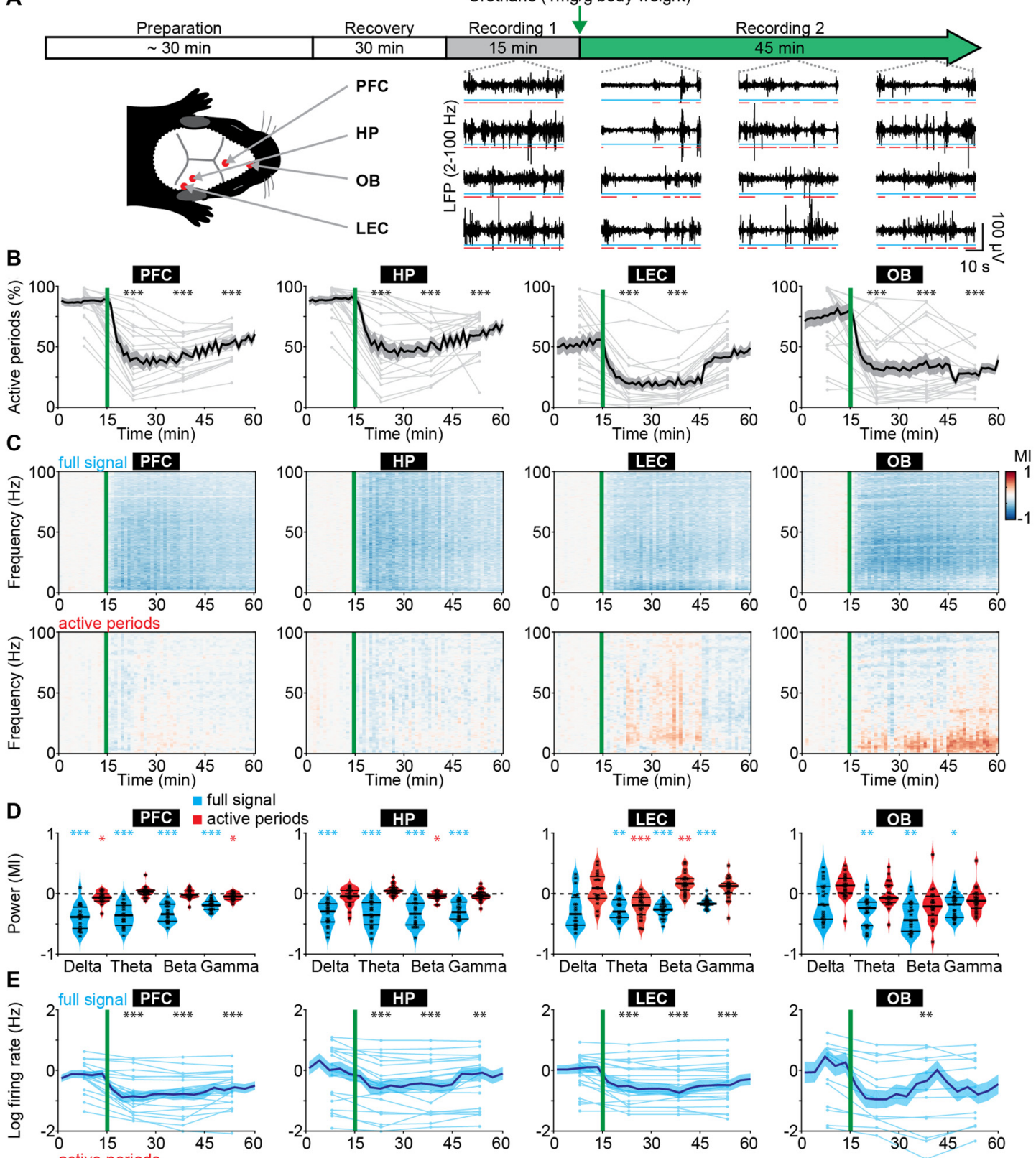

HP

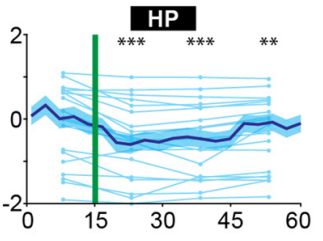

LEC
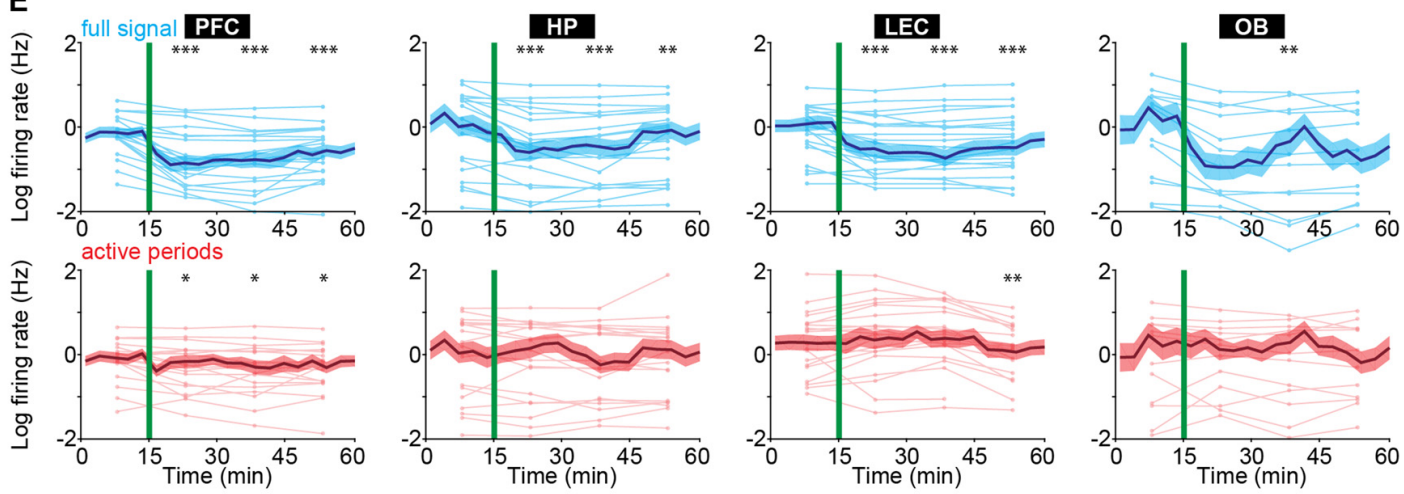

FIGURE 1 | Frequency-unspecific dampening of neuronal activity during urethane anesthesia in neonatal mice. (A) Schematic representation of experimental paradigm and recording sites as well as characteristic local field potential (LFP) recordings of discontinuous activity in the prefrontal cortex (PFC), HP, lateral entorhinal cortex (LEC), and olfactory bulb (OB) of neonatal mice (P8-10) during non-anesthetized and urethane-anesthetized state. Time windows of active periods are marked by red lines. (B) Line plots displaying the relative occurrence of active periods normalized to total recording time in PFC, HP, OB and LEC before and after urethane injection. (C) Color-coded Ml of power spectra for full signal (top) and active periods (bottom) recorded in PFC, HP, LEC and OB of neonatal mice before and after urethane injection. (D) Violin plots displaying the Ml of power in delta (2-4 Hz), theta-alpha (4-12 Hz), beta (12-30 Hz) and gamma (30-100 Hz) frequency bands for full signal (blue) and active periods (red) recorded in the PFC, HP, LEC and OB. (E) Line plots displaying multi-unit activity (MUA) rates during full signal (blue) and active periods (red). In (B,C,E) green lines correspond to the time point of urethane injection. 
and suppressed activity (from here referred to as "silent periods"; Figure 1A; Khazipov et al., 2004; Hanganu et al., 2006; Brockmann et al., 2011; Bitzenhofer et al., 2017; Gretenkord et al., 2019). The prevalence of active periods decreased rapidly and robustly over time in all investigated brain areas upon urethane injection (Figure 1B). The most prominent reduction was observed 5-15 min after urethane injection. A partial recovery towards baseline levels during the following 30 min was detected in cortical areas, and to a lesser extent in OB (Figure 1B). The temporal sequence of events likely reflects the pharmacokinetics of urethane and is line with the previously reported quick onset (few minutes) and long-lasting effects of urethane anesthesia (2-4 h; Huh and Cho, 2013).

The anesthesia-induced reduced occurrence of active periods was reflected in a broadband $(1-100 \mathrm{~Hz})$ decrease in oscillatory power shown as modulation index (MI) defined as ( $_{\text {power }}$ post - power $\left._{\text {pre }}\right) /\left(\right.$ power $_{\text {post }}+$ power $\left._{\text {pre }}\right)$. In contrast, power spectra during active periods were largely unaffected (Figure 1C). Of note, for $\mathrm{OB}$, delta and theta power during active periods increased over time after urethane injection and might be related to an observed augmentation of respiration amplitude. Spectral properties of full signal and active periods were quantified for delta $(2-4 \mathrm{~Hz})$, theta-alpha $(4-12 \mathrm{~Hz})$, beta $(12-30 \mathrm{~Hz})$ and gamma $(30-100 \mathrm{~Hz})$ frequency bands for the first 15 min post urethane administration, the time window of strongest reduction of active periods. In contrast to the significant reduction of full signal power in all frequency bands, the power during active periods was only marginally affected by anesthesia (Figure 1D). Thus, urethane anesthesia affected network activity in the immature rodent brain predominantly by decreasing the amount of active periods without perturbing the frequency structure of active periods. This is in stark contrast with the well-characterized switch from a low-amplitude high-frequency regime to a high-amplitude low-frequency regime of electrical activity that has been reported for the adult rodent and human brain (Voss and Sleigh, 2007; Alkire et al., 2008).

Anesthesia was shown to induce alterations of long-range network interactions in adult rodents (Bettinardi et al., 2015) and humans (Ferrarelli et al., 2010; Lewis et al., 2012; Sarasso et al., 2015). We examined whether similar alterations are present in the immature mouse brain. Simultaneous recordings of HP and PFC, as well as OB and LEC were analyzed to assess the effects of anesthesia on long-range functional coupling. We previously showed that at the end of the first postnatal week hippocampal theta bursts drive the oscillatory entrainment of local circuits in the PFC, whereas discontinuous activity in $\mathrm{OB}$ controls the network activity in LEC (Brockmann et al., 2011; Ahlbeck et al., 2018; Gretenkord et al., 2019). Urethane did not modify these interactions. The synchrony within networks quantified by HP-PFC and OB-LEC coherence was similar during baseline (no urethane anesthesia) and in the presence of urethane (Supplementary Figure S1A). These data indicate that the core features of long-range functional coupling are retained under anesthesia in neonatal mice.

Anesthesia modified neuronal firing in all investigated areas. Firing rates in PFC, HP, LEC and OB decreased after urethane injection and only partially recovered during the following $45 \mathrm{~min}$ (Figure 1E). However, firing rates during active periods were only marginally affected. To examine whether the timing of neuronal firing to the phase of oscillatory activity was altered by anesthesia, we calculated PPC, a firing rate-independent measure of spike-LFP phase locking (Vinck et al., 2010). All four brain regions showed similar frequencyresolved phase locking profiles before and after urethane injection (Supplementary Figures S1B,C).

Anesthetics have been shown to alter the excitation/inhibition balance in the adult brain through their action on specific ion channels involved in synaptic transmission (Gao et al., 2017). Such alteration is usually monitored by changes in the $1 / \mathrm{f}$ slope of power spectral density. Further, signal complexity and information content measured by sample entropy have been correlated with behavioral states of adults, such as consciousness, sleep/wake states and anesthesia (Liang et al., 2015; Liu et al., 2018). For neonatal mice, we observed similar values of $1 / f$ slope and sample entropy before and during urethane anesthesia (Supplementary Figures S1D-F), suggesting that urethane does not perturb cortical excitation/inhibition balance and signal complexity at this early age. The findings provide additional evidence to the hypothesis that anesthesia has unique effects on the immature brain.

To add additional evidence for this hypothesis, we extended the time window of investigation and performed extracellular recordings from the PFC of juvenile mice (P24-39). At this age oscillatory activity is continuous, thus a distinction between active and inactive periods is not possible. In contrast to the frequency-unspecific reduction of active periods in neonates, urethane anesthesia increased the oscillatory power in the delta frequency band and suppressed power in beta and gamma frequency bands (Supplementary Figure S2), confirming the anesthetic effects in the adult brain (Alkire et al., 2008; Chauvette et al., 2011; Purdon et al., 2015).

Taken together, these results indicate that urethane anesthesia dampened neonatal brain activity mainly by augmenting the discontinuity of network activity, i.e., reducing the proportion of time the brain spent in active periods. However, the active periods were largely unaffected in their temporal structure and firing dynamics. In contrast, urethane anesthesia in older mice led to frequency-specific changes. Thus, urethane anesthesia differently impacts neonatal and adult brain activity in mice.

\section{Suppression of Active Periods Predicts Anesthetic Concentration in Neonatal Mice}

To test whether the effects of urethane on neonatal brain activity generalize to other anesthetics, we performed LFP and MUA recordings from $\mathrm{HP}$ and $\mathrm{PFC}$ of $\mathrm{P} 8-10$ mice at increasing doses of isoflurane-induced anesthesia $(0,1,2$ and $3 \% ; 15$ min per concentration; Figure 2A). Urethane hyperpolarizes neurons by potentiating a resting potassium conductance (Pagliardini et al., 2013), whereas most other anesthetics, such as isoflurane and sevoflurane, mainly act by potentiating $\mathrm{GABA}_{\mathrm{A}}$ receptormediated transmission (Franks, 2006). Isoflurane reduced the incidence of active periods in a dose-dependent manner (Figure 2B). Accordingly, the broadband reduction of LFP power 

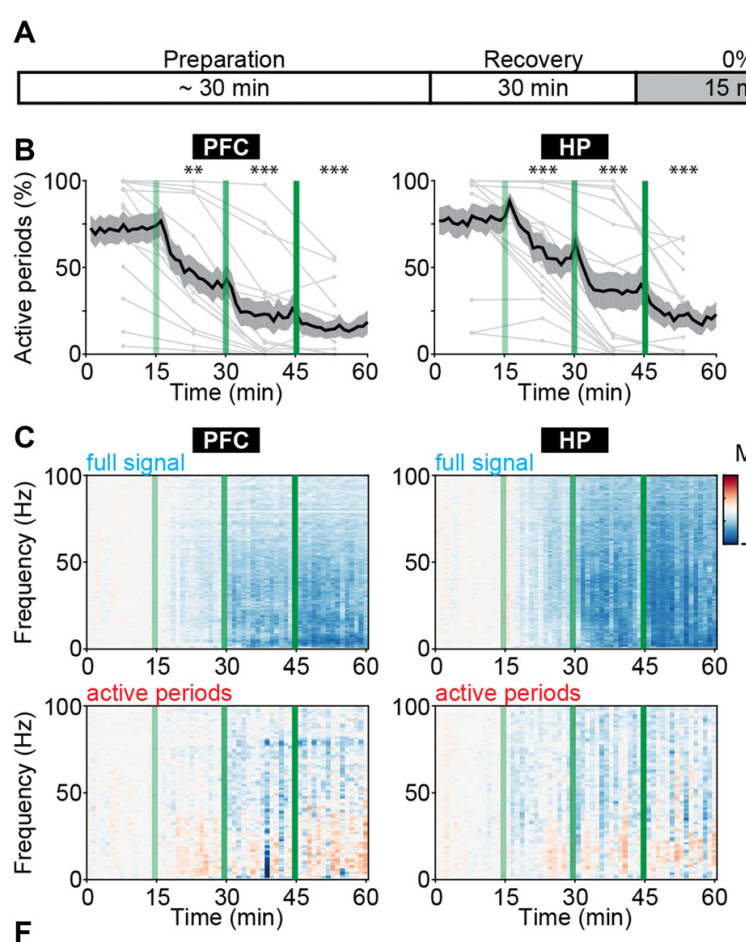

F
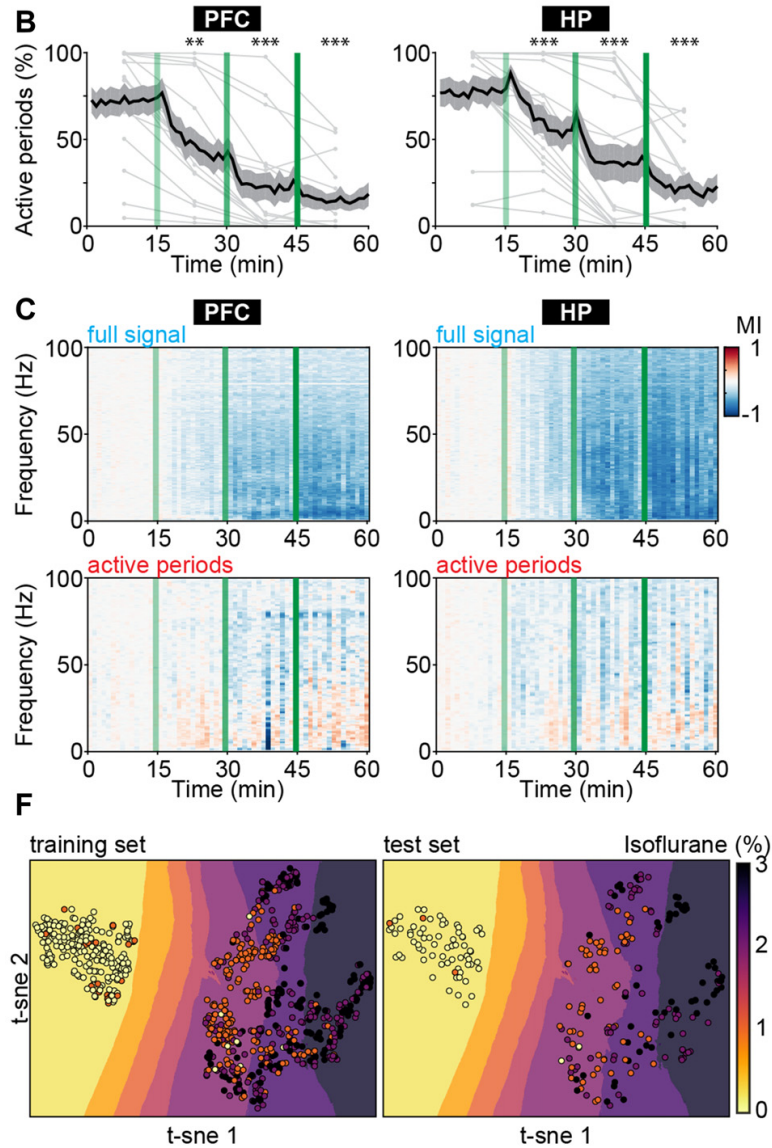

HP

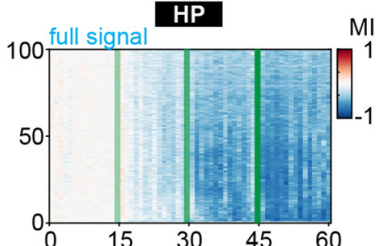

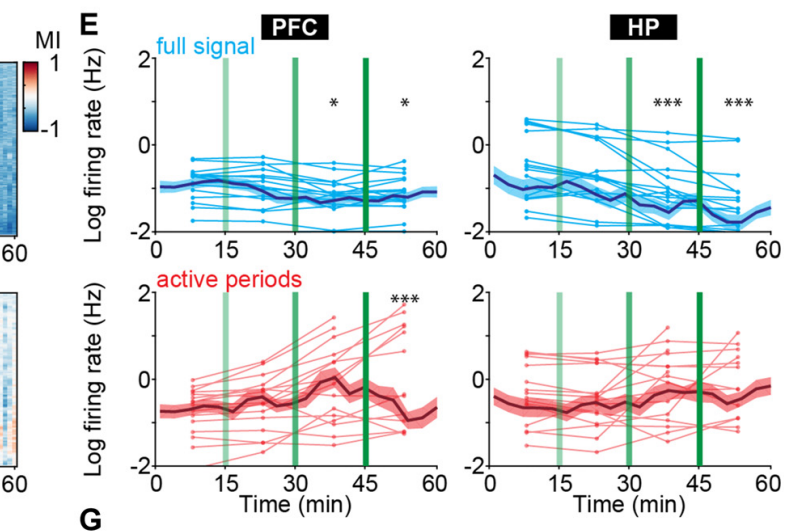

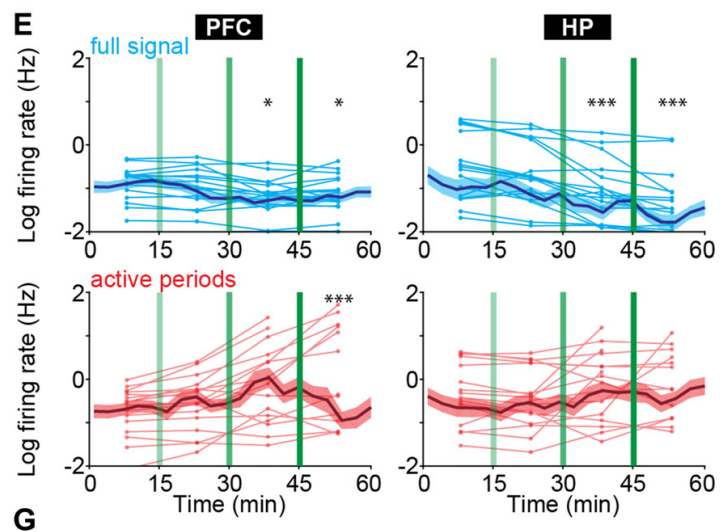

G

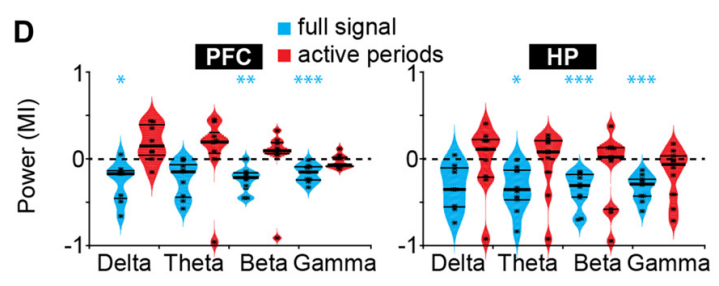

- anesthesia level

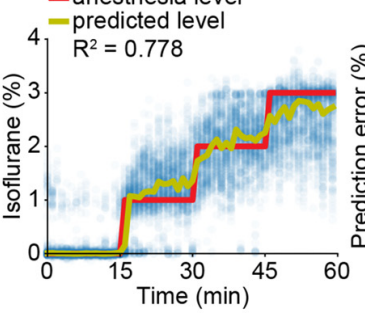

median absolute error $=0.191$

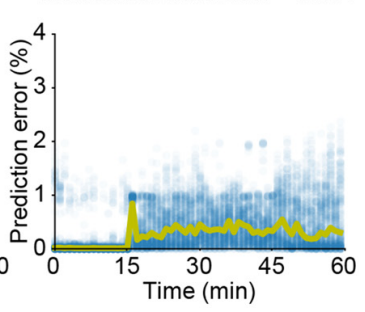

FIGURE 2 | Suppression of active periods in relationship with the depth of isoflurane anesthesia in neonatal mice. (A) Schematic representation of experimental protocol for LFP recordings without anesthesia and during increasing levels of isoflurane anesthesia in neonatal mice (P8-10). (B) Line plots displaying the relative occurrence of active periods in PFC and HP during increasing levels of isoflurane anesthesia. (C) Color-coded MI of power spectra for full signal (top) and active periods (bottom) during increasing levels of isoflurane anesthesia. (D) Violin plots displaying the Ml of power in delta (2-4 Hz), theta (4-12 Hz), beta (12-30 Hz) and gamma $(30-100 \mathrm{~Hz})$ frequency bands for full signal (blue) and active periods (red). (E) Line plots displaying MUA firing rates during full signal (blue) and active periods (red). In (B,C,E) green lines correspond to the time points of increasing isoflurane anesthesia. (F) Visualization of anesthesia depth prediction by t-sne plots. Background color codes for predicted anesthesia depth, while the color of the dots represents the actual anesthesia level in the training (left) and test set (right). (G) Scatter plots displaying anesthesia depth predictions with support vector regression (left) and absolute errors between anesthesia depth prediction and actual anesthesia depth (right).

was also dependent on isoflurane concentration (Figures 2C,D). Power spectra of active periods remained largely unaffected in the presence of isoflurane, similarly to the urethane effects (Figures 2C,D). MUA rates during active periods in PFC and HP were hardly modified in the presence of isoflurane, yet the overall firing decreased corresponding to the reduced occurrence of active periods (Figure 2E). Together, these findings identify the suppression of active periods as the main effect of bolus urethane injection and isoflurane anesthesia in the neonatal mouse brain.

The development-specific response of the immature brain to anesthesia might represent the main obstacle when trying to predict anesthesia depth in infants using algorithms based on the mature brain activity of adults. Therefore, we next aimed to use electrophysiological properties specific for anesthetized neonatal mice to predict the concentration of administered isoflurane. We used support vector regression (Supplementary Figure S3), with the following input features: median amplitude of broadband LFP, percent of time spent in active periods, and spectral power from 1 to $100 \mathrm{~Hz}$ in $10 \mathrm{~Hz}$ bins for both hippocampal and prefrontal activity. An additional feature was the output of a support vector classifier that received the same features as for the support vector regression, and that was designed to predict whether the animal was under anesthesia or not. The algorithm accurately predicted anesthesia depth across all levels of isoflurane concentration (Figures 2F,G). Estimation of information content of the different features identified the median amplitude of broadband LFP as the most informative feature (Supplementary Figure S4A). As the power of active 
periods was only marginally affected by anesthesia, this feature mainly mirrors the suppression of active periods. Interestingly, the algorithm was also able to distinguish non-anesthetized from anesthetized recordings from neonatal mice under urethane, even though it had not been exposed to this dataset during training (Supplementary Figure S4B).

Thus, features of electrophysiological activity that capture the particularities of immature neuronal networks can predict anesthetic concentration in neonatal mice. The generalization of the classifier to a different anesthetic indicates that it can identify general anesthesia-related features of brain activity in neonatal mice.

\section{Frequency-Unspecific Suppression of Activity in Anesthetized Human Neonates and Young Infants}

To test if human neonates and infants, similarly to mice, respond to anesthesia with a broadband decrease of periods of oscillatory activity, we examined EEG recordings from humans aged 0-6 months postnatal age, who received general anesthesia with volatile anesthetics (sevoflurane 32 subjects, isoflurane two subjects, desflurane one subject) for surgery (Supplementary Table S1).

In neonatal mice, the median LFP amplitude of broadband activity was identified as the most informative feature to predict anesthetic depth. We, therefore, applied the same data analysis approach to human EEG data (Supplementary Figure S5). We found the median amplitude of broadband EEG activity (averaged across all recording electrodes across the scalp) was negatively correlated with end-tidal anesthetic concentration (etAnesthetic) in human neonates from birth until 2 months postnatal age (Figures 3A,B). For older human infants, the correlation of the median EEG amplitude with the anesthetic concentration switched to a positive correlation, in agreement with adult human data (Hagihira, 2015). This relationship was even stronger using expected birth age, corrected for conceptional age (Supplementary Figure S6A). This switch from negative to positive correlation was also visible in the normalized median EEG amplitude when averaged for age-grouped babies (0-2, 2-4, 4-6 months; Figure 3C).

Quantification of median EEG amplitude across frequencies revealed a broadband suppression of EEG activity in human neonates of $0-2$ months (Figure 3D). In contrast, the relationship between activity amplitude and etAnesthetic indicated frequency-specificity in human infants of 2-4 and 4-6 months, as previously reported (Cornelissen et al., 2017). Frontal activity has been shown to be particularly sensitive to age-varying anesthesia-related effects in human neonates (Cornelissen et al., 2015). Analysis of only frontal electrodes (Fp1, Fp2, F3, F4, F7, F8, Fpz) showed the same age-dependent anesthesia-induced changes as analysis of full scalp electrodes (Supplementary Figures S6B-D).

Thus, analogous to what we found in neonatal mice, general anesthesia in human infants younger than 2 months suppressed neuronal population activity, as reported previously (Cornelissen et al., 2015), while at older age anesthesia induced frequencyspecific effects.
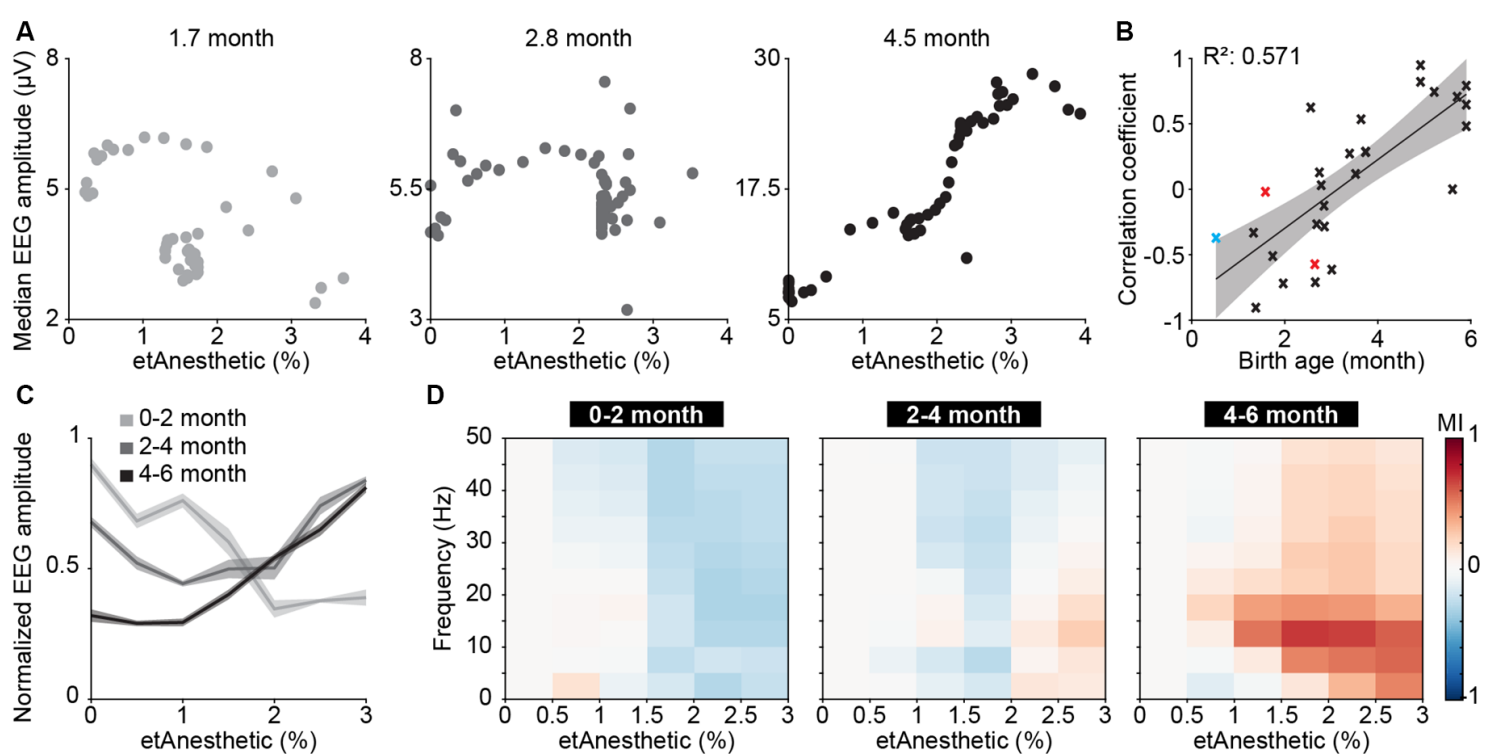

2-4 month
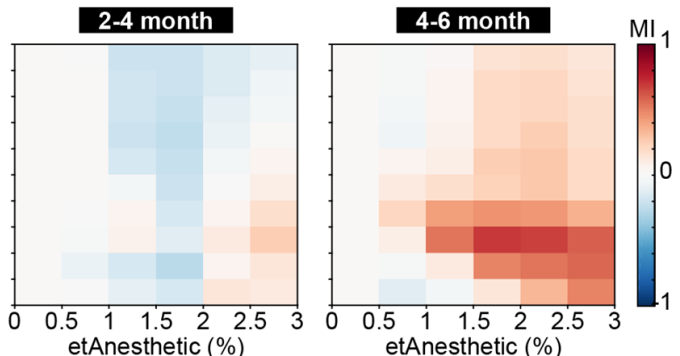

FIGURE 3 | Age-dependent switch from broadband suppression to frequency-specific effects of general anesthesia on electroencephalographic (EEG) activity in human neonates and infants. (A) Scatter plots displaying the median EEG amplitude as a function of anesthetic concentration for representative examples of 0-2, 2-4 and 4-6 months of age. (B) Scatter plot displaying the correlation coefficient of median EEG amplitude and anesthetic concentration in relation to birth age for sevoflurane (black), isoflurane (red), and desflurane (blue). (C) Line plots displaying normalized EEG amplitude as a function of anesthetic concentration. (D) Color-coded Ml of median EEG amplitudes in different frequency bands as a function of anesthetic concentration for human babies of 0-2 months (left), 2-4 months (middle) and 4-6 months (right). 


\section{A Model to Predict End-Tidal Volume of Sevoflurane Anesthesia in Human Neonates and Infants}

The correlation of EEG activity with etAnesthetic as well as the similar effects of anesthesia in neonatal mice and in humans from birth to 2 months old, suggests that anesthetic depth in babies might be predicted using similar features to those used in neonatal mice. To test this, we used a machine-learning algorithm with a similar architecture as the one we developed for neonatal mice (Supplementary Figure S3). The algorithm was modified to account for the developmental switch from broadband suppression to frequency-specific modulation by training three different regressors using 2 and 4 months as cut-offs. All regressors received the same input features (see "Materials and Methods" section and Supplementary Figure S5). Features derived from EEG activity were able to predict etAnesthetic with high accuracy for all age groups (0-2 months $\mathrm{R}^{2}=0.806,2-4$ months $\mathrm{R}^{2}=0.688,4-6$ months $\mathrm{R}^{2}=0.787$; Figures $4 \mathbf{A}-\mathbf{C}$ ). In line with the frequency-specific alterations observed only in the older age groups, frequency-related features were rated more important for prediction of anesthesia depth in infants of 2-4 and 4-6 months than in neonates of $0-2$ months (Supplementary Figures S7A-C). Predicting anesthesia depth for all ages with a single classifier considering age as an input feature performed with high accuracy (0-6 months $\mathrm{R}^{2}=0.689$; Figure 4D, Supplementary Figure S7D). Training the predictor with only $20 \%$ of the data and testing it on the remaining $80 \%$ resulted in a reduced, but still solid prediction $\left(\mathrm{R}^{2}=0.512\right.$, median absolute error $\left.=0.209\right)$ indicating the robustness of the predictor (Supplementary Figure S8A). This result confirms the age-varying effects of anesthesia on the brain and stresses the importance of considering age when developing algorithms aiming to assess anesthetic depth. Predicting anesthesia depth with a reduced number of four EEG channels (F4, P4, F3, P3) or two EEG channels (P4, P3) often used for long-term monitoring yielded similar results (four channels $\mathrm{R}^{2}=0.713$, median absolute error $=0.134$; two channels $\mathrm{R}^{2}=0.671$, median absolute error $=0.161$; Supplementary Figures S8B,C).

Thus, mouse and human neonates show similar changes in network activity in response to anesthesia. These results highlight how neurophysiological activity could be beneficial for future attempts at predicting anesthetic depth in clinical settings.

\section{DISCUSSION}

Monitoring brain function during anesthesia is desirable to avoid intraoperative awareness and side effects resulting from unnecessarily high doses of anesthetics. Since consciousness is an elusive concept and cannot be directly measured, EEG features have been used to guide anesthesia delivery during human surgery. Monitoring methods developed for adults perform poorly in human neonates and infants, particularly during the first months of life (Davidson et al., 2005; Hayashi et al., 2012; Poorun et al., 2016; Koch et al., 2017). Age-specific effects of anesthetics on immature brain activity are considered

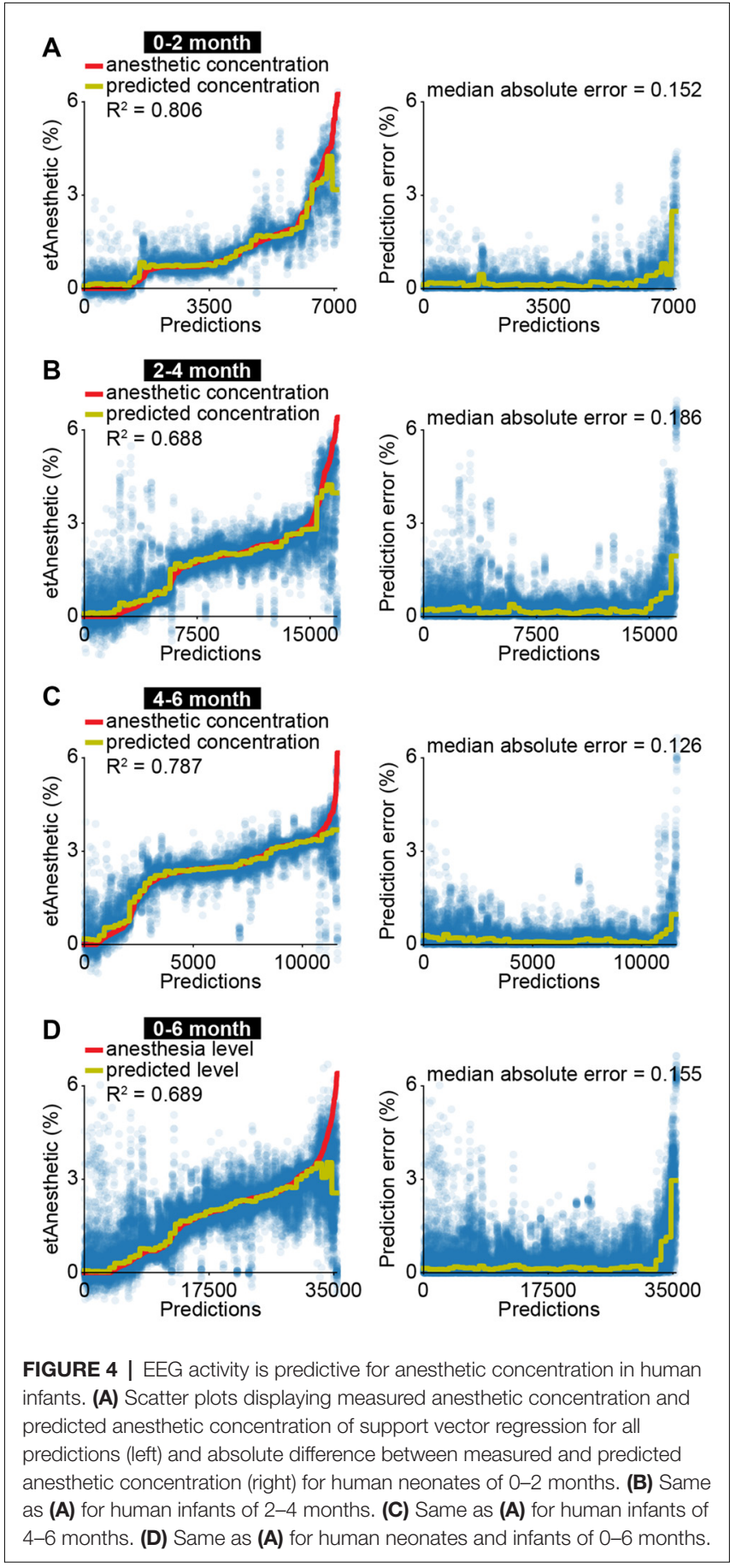

the main reason for such poor performance. Implementation of neonate- and infant-specific anesthesia monitors requires elucidation of distinct anesthesia-induced EEG features during early development. We took advantage of a translational approach to address this open question. We first carried out an in-depth investigation of anesthetic effects on brain activity in neonatal mice, and then applied this knowledge to develop features that would correlate with an anesthetic concentration in human neonates. 
In contrast to the continuous EEG signal observed in adults, neonatal EEG around birth is characterized by a highly discontinuous and fragmented temporal organization, with bursts of cerebral activity (active periods) alternating with interburst intervals lacking activity (silent periods; Anderson et al., 1985; Connell et al., 1987; Stockard-Pope et al., 1992; Lamblin et al., 1999; Vecchierini et al., 2003, 2007; Stevenson et al., 2017). Neonatal mice show a similar discontinuous organization of cortical activity (Khazipov et al., 2004; Hanganu et al., 2006; Brockmann et al., 2011). In accordance with the similar organization of early activity patterns in age-matched mouse pups and human infants, we found comparable effects of anesthesia on LFP and EEG signals, respectively.

It is well established that in the adult rodent and human brain most anesthetics favor slow oscillations at the expense of faster ones, thereby slowing the EEG rhythm (Alkire et al., 2008; Chauvette et al., 2011; Purdon et al., 2015). This principle is thought to underlie most algorithms that are clinically used to predict anesthesia depth (Davidson et al., 2005). Indeed, such algorithms perform poorly with anesthetics, such as ketamine, that do not share this mechanism of action (Hans et al., 2005). In line with previous studies (Ackman et al., 2014; Kirmse et al., 2015), we report that both urethane and isoflurane anesthesia affect brain activity in a different way in neonatal mice. Instead of favoring slow oscillations at the expense of faster ones, anesthesia in neonatal mice broadly suppresses activity in a frequencyunspecific manner. The dampening of cortical activity for human infants of 0-2 months suggests a development specific effect of anesthesia on immature brain activity that translates between mice and humans.

In rodents, the switch from activity suppression to frequencyspecific modulation of neuronal activity by anesthesia has been reported to occur around P12 (Ackman et al., 2014). This coincides with the emergence of slow oscillations during sleep, suggested to depend on the maturation of thalamocortical networks (Steriade et al., 1993; Ackman et al., 2014). Consistent with our previous studies evaluating EEG properties of this data set, we found that theta and alpha oscillatory activity under anesthesia emerges in humans at around 4 months postnatal age (Cornelissen et al., 2015, 2017, 2018a). Developmental changes in the brain explain the changes in EEG dynamics that occur with postnatal age. In humans, gross brain development occurs in a caudal to rostral direction, with myelination of the medulla, pons, and thalamus starting within the first few postnatal weeks, and frontal cortex myelination starting around 3-4 postnatal months (Brody et al., 1987; Kinney et al., 1988). Regional differences in the rate of synaptogenesis, glucose metabolism and myelination across the cortex occur between subcortical and cortical regions, and between different regions of the cortex during the first 12 postnatal months in human infants (Huttenlocher and Dabholkar, 1997; Tau and Peterson, 2010; Dehorter et al., 2012; Catts et al., 2013; Semple et al., 2013; Murata and Colonnese, 2019). A key role in brain development is played by subplate neurons, the first neurons generated in the cerebral cortex, which guide the formation of thalamocortical connections (Kanold and Luhmann, 2010; Kostović and Judas, 2010). The subplate cells form the first functional connections and relay oscillatory activity in the developing brain (Kanold and Luhmann, 2010). Alpha oscillations in the anesthetized brain are postulated to be produced by thalamocortical circuits, and the gradual emergence of highly powered alpha oscillations at 4-months of age suggests an important developmental milestone has been reached in the processes guided by the subplate neurons. Future studies with a wider age range in mice and humans, including data of human infants studied at preterm, and children in older than 6 months of age, may deepen the understanding of anesthetic effects on brain activity throughout development.

The anesthetics evaluated across species in this study were comparable but not identical in terms of mechanism of action. Moreover, anesthetic management practices used in mice were simplified compared to commonly-used anesthetic practices in the clinic. Multimodal anesthesia requires the use of low-dose anesthetics in combination with analgesic and neuromuscular blocking agents to provide optimal anesthesia and reduce the side effect. These agents act on different drug targets in the nervous system and may have subtle but different effects on brain oscillatory activity (Brown et al., 2018).

In adult human volunteers, the correlation with anesthetic depth and EEG parameters can be performed using verbal reports to establish a threshold for unconsciousness (Purdon et al., 2015). However, in non-verbal populations such as human infants, one must rely on indirect behavioral measures which are more readily performed on emergence rather than induction and incision (Cornelissen et al., 2018b). Future investigations need to include surgical incision and other stimuli into the mouse models to understand with greater granularity the anesthetic titration around the minimal concentrations required to suppress movement, autonomic, and cortical responses to noxious stimuli.

In summary, we report that the suppression of brain activity in mouse and human neonates correlates with anesthetic concentration. The detailed understanding of anesthesia effects on network activity in mice allowed us to identify features and develop a machine-learning algorithm that is able to predict anesthetic concentration from EEG recordings in human neonates. We propose that, after appropriate training, an algorithm based on what we introduce here could learn to associate specific EEG effects with certain anesthetic doses. Eventual mismatches between administered and predicted anesthetic dose would then identify patients that are particularly sensitive/insensitive to an anesthetic, thus helping the anesthetist in administering appropriate levels of anesthetics. By these means, the risk of adverse neurodevelopmental outcome might be mitigated.

\section{ETHICS STATEMENT}

All animal experiments were performed in compliance with the German laws and the guidelines of the European Community for the use of animals in research and were approved by the local ethical committee (G132/12, G17/015, N18/015). Human neonates and infants who were scheduled for an elective surgical procedure were recruited from the pre-operative clinic at Boston Children's Hospital from 12/2012 to 08/2018 (under Institutional 
Review Board P-3544, with written informed consent obtained from parents/legal guardians).

\section{AUTHOR CONTRIBUTIONS}

MC, SB and IH-O designed the experiments. MC, SB, SG, JK, $\mathrm{JP}$, and LC carried out the experiments. MC, SB, SG, JK and JP analyzed the data. MC, SB, LC, CB and IH-O interpreted the data and wrote the article. All authors discussed and commented on the manuscript.

\section{FUNDING}

This work was funded by grants from the European Research Council (ERC-2015-CoG 681577 to IH-O), the German Research

\section{REFERENCES}

Ackman, J. B., Zeng, H., and Crair, M. C. (2014). Structured dynamics of neural activity across developing neocortex. BioRxiv [Preprint]. doi: 10.1101/012237

Ahlbeck, J., Song, L., Chini, M., Bitzenhofer, S. H., and Hanganu-Opatz, I. L. (2018). Glutamatergic drive along the septo-temporal axis of hippocampus boosts prelimbic oscillations in the neonatal mouse. Elife 7:e33158. doi: $10.7554 /$ eLife.33158

Alkire, M. T., Hudetz, A. G., and Tononi, G. (2008). Consciousness and anesthesia. Science 322, 876-880. doi: 10.1126/science.1149213

Anderson, C. M., Torres, F., and Faoro, A. (1985). The EEG of the early premature. Electroencephalogr. Clin. Neurophysiol. 60, 95-105. doi: 10.1016/00134694(85)90015-X

Bettinardi, R. G., Tort-Colet, N., Ruiz-Mejias, M., Sanchez-Vives, M. V., and Deco, G. (2015). Gradual emergence of spontaneous correlated brain activity during fading of general anesthesia in rats: evidences from fMRI and local field potentials. Neuroimage 114, 185-198. doi: 10.1016/j.neuroimage.2015. 03.037

Bitzenhofer, S. H., Ahlbeck, J., Wolff, A., Wiegert, J. S., Gee, C. E., Oertner, T. G., et al. (2017). Layer-specific optogenetic activation of pyramidal neurons causes beta-gamma entrainment of neonatal networks. Nat. Commun. 8:14563. doi: $10.1038 /$ ncomms 14563

Brockmann, M. D., Pöschel, B., Cichon, N., and Hanganu-Opatz, I. L. (2011). Coupled oscillations mediate directed interactions between prefrontal cortex and hippocampus of the neonatal rat. Neuron 71, 332-347. doi: 10.1016/j. neuron.2011.05.041

Brody, B. A., Kinney, H. C., Kloman, A. S., and Gilles, F. H. (1987). Sequence of central-nervous-system myelination in human infancy: I. An autopsy study of myelination. J. Neuropath. Exp. Neurol. 46, 283-301. doi: 10.1097/00005072198705000-00005

Brown, E. N., Pavone, K. J., and Naranjo, M. (2018). Multimodal general anesthesia: theory and practice. Anesth. Analg. 127, 1246-1258. doi: 10.1213/ANE.0000000000003668

Catts, V. S., Fung, S. J., Long, L. E., Joshi, D., Vercammen, A., Allen, K. M., et al. (2013). Rethinking schizophrenia in the context of normal neurodevelopment. Front. Cell. Neurosci. 7:60. doi: 10.3389/fncel.2013.00060

Chang, P. S., Walker, S. M., and Fitzgerald, M. (2016). Differential suppression of spontaneous and noxious-evoked somatosensory cortical activity by isoflurane in the neonatal rat. Anesthesiology 124, 885-898. doi: 10.1097/ALN. 0000000000001017

Chauvette, S., Crochet, S., Volgushev, M., and Timofeev, I. (2011). Properties of slow oscillation during slow-wave sleep and anesthesia in cats. J. Neurosci. 31, 14998-15008. doi: 10.1523/JNEUROSCI.2339-11.2011

Cichon, N. B., Denker, M., Grün, S., and Hanganu-Opatz, I. L. (2014). Unsupervised classification of neocortical activity patterns in neonatal and pre-juvenile rodents. Front. Neural Circuits 8:50. doi: 10.3389/fncir.2014.00050
Foundation (Ha 4466/10-1, SPP 1665, SFB 936 B5 to IH-O) and the International Anesthesia Research Society (to LC). IH-O is a founding member of FENS Kavli Network of Excellence. This manuscript has been released as a Pre-Print at BioRxiv.

\section{ACKNOWLEDGMENTS}

We thank P. Putthoff, A. Marquardt, A. Dahlmann, and K. Titze for excellent technical assistance.

\section{SUPPLEMENTARY MATERIAL}

The Supplementary Material for this article can be found online at: https://www.frontiersin.org/articles/10.3389/fncir.2019.000 38/full\#supplementary-material

Colonnese, M. T., and Khazipov, R. (2010). "Slow activity transients" in infant rat visual cortex: a spreading synchronous oscillation patterned by retinal waves. J. Neurosci. 30, 4325-4337. doi: 10.1523/JNEUROSCI.499509.2010

Connell, J. A., Oozeer, R., and Dubowitz, V. (1987). Continuous 4-channel EEG monitoring: a guide to interpretation, with normal values, in preterm infants. Neuropediatrics 18, 138-145. doi: 10.1055/s-2008-1052466

Cornelissen, L., Bergin, A. M., Lobo, K., Donado, C., Soul, J. S., and Berde, C. B. (2017). Electroencephalographic discontinuity during sevoflurane anesthesia in infants and children. Paediatr. Anaesth. 27, 251-262. doi: 10.1111/pan. 13061

Cornelissen, L., Donado, C., Lee, J. M., Liang, N. E., Mills, I., Tou, A., et al. (2018a). Clinical signs and electroencephalographic patterns of emergence from sevoflurane anaesthesia in children: an observational study. Eur. J. Anaesthesiol. 35, 49-59. doi: 10.1097/EJA.0000000000000739

Cornelissen, L., Kim, S. E., Lee, J. M., Brown, E. N., Purdon, P. L., and Berde, C. B. (2018b). Electroencephalographic markers of brain development during sevoflurane anaesthesia in children up to 3 years old. Br. J. Anaesth. 120, 1274-1286. doi: 10.1016/j.bja.2018.01.037

Cornelissen, L., Kim, S. E., Purdon, P. L., Brown, E. N., and Berde, C. B. (2015). Age-dependent electroencephalogram (EEG) patterns during sevoflurane general anesthesia in infants. Elife 4:e06513. doi: 10.7554/eLife 06513

Davidson, A. J., Huang, G. H., Rebmann, C. S., and Ellery, C. (2005). Performance of entropy and bispectral index as measures of anaesthesia effect in children of different ages. Br. J. Anaesth. 95, 674-679. doi: 10.1093/bja/ aei247

Dehorter, N., Vinay, L., Hammond, C., and Ben-Ari, Y. (2012). Timing of developmental sequences in different brain structures: physiological and pathological implications. Eur. J. Neurosci. 35, 1846-1856. doi: 10.1111/j.14609568.2012.08152.x

Ferrarelli, F., Massimini, M., Sarasso, S., Casali, A., Riedner, B. A., Angelini, G., et al. (2010). Breakdown in cortical effective connectivity during midazolaminduced loss of consciousness. Proc. Natl. Acad. Sci. U S A 107, 2681-2686. doi: 10.1073/pnas.0913008107

Franks, N. P. (2006). Molecular targets underlying general anaesthesia. $\mathrm{Br}$. J. Pharmacol. 147, S72-S81. doi: 10.1038/sj.bjp.0706441

Gao, R., Peterson, E. J., and Voytek, B. (2017). Inferring synaptic excitation/inhibition balance from field potentials. Neuroimage 158, 70-78. doi: 10.1016/j.neuroimage.2017.06.078

Glass, P. S., Bloom, M., Kearse, L., Rosow, C., Sebel, P., and Manberg, P. (1997). Bispectral analysis measures sedation and memory effects of propofol, midazolam, isoflurane and alfentanil in healthy volunteers. Anesthesiology 86, 836-847. doi: 10.1097/00000542-199704000-00014

Gretenkord, S., Kostka, J. K., Hartung, H., Watznauer, K., Fleck, D., MinierToribio, A., et al. (2019). Coordinated electrical activity in the olfactory bulb 
gates the oscillatory entrainment of entorhinal networks in neonatal mice. PLoS. Biol. 17:e2006994. doi: 10.1371/journal.pbio.2006994

Hagihira, S. (2015). Changes in the electroencephalogram during anaesthesia and their physiological basis. Br. J. Anaesth. 115, i27-i31. doi: 10.1093/bja/ aev212

Hajat, Z., Ahmad, N., and Andrzejowski, J. (2017). The role and limitations of EEG-based depth of anaesthesia monitoring in theatres and intensive care. Anaesthesia 72, 38-47. doi: 10.1111/anae.13739

Hanganu, I. L., Ben-Ari, Y., and Khazipov, R. (2006). Retinal waves trigger spindle bursts in the neonatal rat visual cortex. J. Neurosci. 26, 6728-6736. doi: 10.1523/JNEUROSCI.0752-06.2006

Hans, P., Dewandre, P. Y., Brichant, J. F., and Bonhomme, V. (2005). Comparative effects of ketamine on bispectral index and spectral entropy of the electroencephalogram under sevoflurane anaesthesia. Br. J. Anaesth. 94, 336-340. doi: 10.1093/bja/aei047

Hayashi, K., Shigemi, K., and Sawa, T. (2012). Neonatal electroencephalography shows low sensitivity to anesthesia. Neurosci. Lett. 517, 87-91. doi: 10.1016/j. neulet.2012.04.028

Huh, Y., and Cho, J. (2013). Urethane anesthesia depresses activities of thalamocortical neurons and alters its response to nociception in terms of dual firing modes. Front. Behav. Neurosci. 7:141. doi: 10.3389/fnbeh.2013. 00141

Huttenlocher, P. R., and Dabholkar, A. S. (1997). Regional differences in synaptogenesis in human cerebral cortex. J. Comp. Neurol. 387, 167-178. doi: 10.1002/(sici)1096-9861(19971020)387:2<167::aid-cne1 > 3.0.co;2-z

Kalkman, C. J., Peelen, L. M., and Moons, K. G. (2011). Pick up the pieces: depth of anesthesia and long-term mortality. Anesthesiology 114, 485-487. doi: 10.1097/ALN.0b013e31820c2ba2

Kanold, P. O., and Luhmann, H. J. (2010). The subplate and early cortical circuits. Annu. Rev. Neurosci. 33, 23-48. doi: 10.1146/annurev-neuro-060909153244

Kertai, M. D., Whitlock, E. L., and Avidan, M. S. (2012). Brain monitoring with electroencephalography and the electroencephalogram-derived bispectral index during cardiac surgery. Anesth. Analg. 114, 533-546. doi: 10.1213/ANE. 0b013e31823ee030

Khazipov, R., Sirota, A., Leinekugel, X., Holmes, G. L., Ben-Ari, Y., and Buzsaki, G. (2004). Early motor activity drives spindle bursts in the developing somatosensory cortex. Nature 432, 758-761. doi: 10.1038/ nature 03132

Kinney, H. C., Brody, B. A., Kloman, A. S., and Gilles, F. H. (1988). Sequence of Central Nervous-System Myelination in Human Infancy .2. Patterns of Myelination in Autopsied Infants. J. Neuropathol. Exp. Neurol. 47, 217-234. doi: 10.1097/00005072-198805000-00003

Kirmse, K., Kummer, M., Kovalchuk, Y., Witte, O. W., Garaschuk, O., and Holthoff, K. (2015). GABA depolarizes immature neurons and inhibits network activity in the neonatal neocortex in vivo. Nat. Commun. 6:7750. doi: $10.1038 /$ ncomms 8750

Koch, S., Stegherr, A. M., Morgeli, R., Kramer, S., Toubekis, E., Lichtner, G., et al. (2017). Electroencephalogram dynamics in children during different levels of anaesthetic depth. Clin. Neurophysiol. 128, 2014-2021. doi: 10.1016/j.clinph. 2017.07.417

Kostović, I., and Judas, M. (2010). The development of the subplate and thalamocortical connections in the human foetal brain. Acta Paediatr. 99, 1119-1127. doi: 10.1111/j.1651-2227.2010.01811.x

Kreuzer, M. (2017). EEG based monitoring of general anesthesia: taking the next steps. Front. Comput. Neurosci. 11:56. doi: 10.3389/fncom.2017. 00056

Lamblin, M. D., André, M., Challamel, M. J., Curzi-Dascalova, L., d'Allest, A. M., De Giovanni, E., et al. (1999). Electroencephalography of the premature and term newborn. Maturational aspects and glossary. Neurophysiol. Clin. 29, 123-219. doi: 10.1016/S0987-7053(99)80051-3

Lewis, L. D., Weiner, V. S., Mukamel, E. A., Donoghue, J. A., Eskandar, E. N., Madsen, J. R., et al. (2012). Rapid fragmentation of neuronal networks at the onset of propofol-induced unconsciousness. Proc. Natl. Acad. Sci. U S A 109, E3377-E3386. doi: 10.1073/pnas.1210907109

Liang, Z., Wang, Y., Sun, X., Li, D., Voss, L. J., Sleigh, J. W., et al. (2015). EEG entropy measures in anesthesia. Front. Comput. Neurosci. 9:16. doi: $10.3389 /$ fncom. 2015.00016
Liu, Q., Ma, L., Fan, S. Z., Abbod, M. F., and Shieh, J. S. (2018). Sample entropy analysis for the estimating depth of anaesthesia through human EEG signal at different levels of unconsciousness during surgeries. PeerJ 6:e4817. doi: $10.7717 /$ peerj. 4817

Lo, S. S., Sobol, J. B., Mallavaram, N., Carson, M., Chang, C., Grieve, P. G., et al. (2009). Anesthetic-specific electroencephalographic patterns during emergence from sevoflurane and isoflurane in infants and children. Paediatr. Anaesth. 19, 1157-1165. doi: 10.1111/j.1460-9592.2009. 03128.x

Migut, M. A., Worring, M., and Veenman, C. J. (2015). Visualizing multidimensional decision boundaries in 2D. Data Min. Knowl. Discov. 29, 273-295. doi: $10.1007 /$ s10618-013-0342-x

Milh, M., Kaminska, A., Huon, C., Lapillonne, A., Ben-Ari, Y., and Khazipov, R. (2007). Rapid cortical oscillations and early motor activity in premature human neonate. Cereb. Cortex 17, 1582-1594. doi: 10.1093/cercor/ bhl069

Murata, Y., and Colonnese, M. T. (2019). Thalamic inhibitory circuits and network activity development. Brain Res. 1706, 13-23. doi: 10.1016/j.brainres.2018. 10.024

Nolte, G., Bai, O., Wheaton, L., Mari, Z., Vorbach, S., and Hallett, M. (2004). Identifying true brain interaction from EEG data using the imaginary part of coherency. Clin. Neurophysiol. 115, 2292-2307. doi: 10.1016/j.clinph.2004. 04.029

O’Toole, J. M., Boylan, G. B., Vanhatalo, S., and Stevenson, N. J. (2016). Estimating functional brain maturity in very and extremely preterm neonates using automated analysis of the electroencephalogram. Clin. Neurophysiol. 127, 2910-2918. doi: 10.1016/j.clinph.2016.02.024

Pagliardini, S., Funk, G. D., and Dickson, C. T. (2013). Breathing and brain state: urethane anesthesia as a model for natural sleep. Respir. Physiol. Neurobiol. 188, 324-332. doi: 10.1016/j.resp.2013.05.035

Poorun, R., Hartley, C., Goksan, S., Worley, A., Boyd, S., Cornelissen, L., et al. (2016). Electroencephalography during general anaesthesia differs between term-born and premature-born children. Clin. Neurophysiol. 127, 1216-1222. doi: 10.1016/j.clinph.2015.10.041

Prichep, L. S., Gugino, L. D., John, E. R., Chabot, R. J., Howard, B., Merkin, H., et al. (2004). The patient state index as an indicator of the level of hypnosis under general anaesthesia. Br. J. Anaesth. 92, 393-399. doi: 10.1093/bja/ aeh082

Punjasawadwong, Y., Phongchiewboon, A., and Bunchungmongkol, N. (2014). Bispectral index for improving anaesthetic delivery and postoperative recovery. Cochrane Database Syst. Rev. 6:CD003843. doi: 10.1002/14651858. CD003843

Purdon, P. L., Sampson, A., Pavone, K. J., and Brown, E. N. (2015). Clinical electroencephalography for anesthesiologists: Part I: background and basic signatures. Anesthesiology 123, 937-960. doi: 10.1097/ALN.00000000000 00841.

Sarasso, S., Boly, M., Napolitani, M., Gosseries, O., Charland-Verville, V., Casarotto, S., et al. (2015). Consciousness and complexity during unresponsiveness induced by propofol, Xenon and Ketamine. Curr. Biol. 25, 3099-3105. doi: 10.1016/j.cub.2015.10.014

Semple, B. D., Blomgren, K., Gimlin, K., Ferriero, D. M., and NobleHaeusslein, L. J. (2013). Brain development in rodents and humans: identifying benchmarks of maturation and vulnerability to injury across species. Prog. Neurobiol. 106-107, 1-16. doi: 10.1016/j.pneurobio.2013. 04.001

Steriade, M., Nuñez, A., and Amzica, F. (1993). Intracellular analysis of relations between the slow $(<1 \mathrm{~Hz})$ neocortical oscillation and other sleep rhythms of the electroencephalogram. J. Neurosci. 13, 3266-3283. doi: 10.1523/jneurosci. 13-08-03266.1993

Stevenson, N. J., Oberdorfer, L., Koolen, N., O’Toole, J. M., Werther, T., Klebermass-Schrehof, K., et al. (2017). Functional maturation in preterm infants measured by serial recording of cortical activity. Sci. Rep. 7:12969. doi: $10.1038 / s 41598-017-13537-3$

Stockard-Pope, J. E., Werner, S. S., and Bickford, R. G. (1992). Atlas of Neonatal Electroencephalography. New York, NY: Raven Press.

Stolwijk, L. J., Weeke, L. C., de Vries, L. S., van Herwaarden, M. Y. A., van der Zee, D. C., van der Werff, D. B. M., et al. (2017). Effect of general anesthesia on neonatal aEEG-A cohort study of patients with non-cardiac 
congenital anomalies. PLoS One 12:e0183581. doi: 10.1371/journal.pone. 0183581

Tau, G. Z., and Peterson, B. S. (2010). Normal development of brain circuits. Neuropsychopharmacology 35, 147-168. doi: 10.1038/npp.2009.115

Vecchierini, M. F., André, M., and d'Allest, A. M. (2007). Normal EEG of premature infants born between 24 and 30 weeks gestational age: terminology, definitions and maturation aspects. Neurophysiol. Clin. 37, 311-323. doi: 10.1016/j.neucli.2007.10.008

Vecchierini, M. F., d'Allest, A. M., and Verpillat, P. (2003). EEG patterns in 10 extreme premature neonates with normal neurological outcome: qualitative and quantitative data. Brain Dev. 25, 330-337. doi: 10.1016/s03877604(03)00007-x

Vinck, M., van Wingerden, M., Womelsdorf, T., Fries, P., and Pennartz, C. M. (2010). The pairwise phase consistency: a bias-free measure of rhythmic neuronal synchronization. Neuroimage 51, 112-122. doi: 10.1016/j. neuroimage.2010.01.073
Voss, L., and Sleigh, J. (2007). Monitoring consciousness: the current status of EEG-based depth of anaesthesia monitors. Best Pract. Res. Clin. Anaesthesiol. 21, 313-325. doi: 10.1016/j.bpa.2007.04.003

Conflict of Interest Statement: The authors declare that the research was conducted in the absence of any commercial or financial relationships that could be construed as a potential conflict of interest.

Copyright (C) 2019 Chini, Gretenkord, Kostka, Pöpplau, Cornelissen, Berde, Hanganu-Opatz and Bitzenhofer. This is an open-access article distributed under the terms of the Creative Commons Attribution License (CC BY). The use, distribution or reproduction in other forums is permitted, provided the original author(s) and the copyright owner(s) are credited and that the original publication in this journal is cited, in accordance with accepted academic practice. No use, distribution or reproduction is permitted which does not comply with these terms. 